\title{
O Teste de Compreensão Emocional (TEC): Estudos Psicométricos numa População Portuguesa
}

\author{
Maria da Gloria Franco ${ }^{1}$ \\ António Roazzi $i^{2}$ \\ Natalie Nóbrega Santos ${ }^{3}$ \\ ${ }^{1}$ Faculdade de Artes e Humanidades da Universidade da Madeira \\ ${ }^{2}$ Universidade Federal de Pernambuco \\ ${ }^{3}$ Instituto Universitário
}

\begin{abstract}
Resumo
O objetivo deste estudo é apresentar as vantagens e os contributos em contexto escolar do Teste de Compreensão Emocional (TEC) para a população portuguesa, desenvolvido a partir da versão brasileira, que tem por base o instrumento desenvolvido por Harris, Pons e seus colaboradores. No nosso estudo participaram 766 crianças (381 raparigas), com idades compreendidas entre 3 e 13 anos $(M=7.21, D P=1.95)$, de diferentes extratos socioeconómicos. Os resultados mostram que o instrumento tem consistência interna aceitável e que é possível isolar as diferentes fases de desenvolvimento emocional propostas pelos autores. Os componentes que medem relacionam-se com diferentes variáveis importantes em contexto escolar (idade, estatuto socioeconómico, inteligência fluída), e explicam uma parte do rendimento académico e da retenção escolar.

Palavras-chave: inteligência emocional, desenvolvimento emocional, rendimento académico, ensino fundamental, psicometria
\end{abstract}

\section{Abstract}

The Test of Emotional Comprehension (TEC): Psychometric Studies in a Portuguese Population

The aim of this study was to present the advantages and contributions of the Emotional Comprehension Test (ECT) - Brazilian version, based on the instrument developed by Harris, Pons et al., in the school context of the Portuguese population. The sample comprises 766 children (381 girls), aged between 3 to 13 years $(M=7.21, \mathrm{SD}=1.95)$, from different socioeconomic backgrounds. The results show that the instrument has acceptable internal consistency and that it is possible to identify the different stages of emotional understanding proposed by the authors. The components measured relate to different important variables in school context (age, socioeconomic status, fluid intelligence), and explain part of the academic performance and school retention.

Keywords: emotional intelligence; emotional development; academic performance; elementary education; psychometrics

\section{Resumen}

Test de Comprensión Emocional (TEC): Estudios psicométricos en una población portuguesa

El objetivo de este estudio fue presentar ventajas y aportes en el contexto escolar del Test de Comprensión Emocional (TEC) en la población portuguesa, desarrollado a partir de la versión brasileña, que tiene por base el instrumento desarrollado por Harris, Pons y colaboradores. En nuestro estudio participaron 766 alumnos (381 niñas) con edades comprendidas entre los 3 y 13 años $(M=7.21, D T=1.95)$, de diferentes extractos socioeconómicos. Los resultados muestran que el instrumento tiene consistencia interna aceptable y que es posible separar las diferentes etapas de desarrollo emocional propuesta por los autores. Los componentes de medición se relacionan con diferentes variables importantes del contexto escolar (edad, estatuto socioeconómico, inteligencia fluida) y explican una parte del rendimiento académico y de la retención escolar.

Palabras clave: inteligencia emocional; desarrollo emocional; rendimiento académico; Enseñanza Primaria; psicometría

A compreensão emocional (CE) é um conceito desenvolvimental, que tem sido estudado junto com o conhecimento emocional, a inteligência emocional ou regulação emocional, ora como sendo sinónimo de uns, ora como sendo uma parte de outros, ora como tendo uma individualidade própria (Djambazova-Popordanoska, 2016; Franco \& Santos, 2015; Roazzi, et al., 2015). Em 2004, Pons, Harris, e Rosnay propuseram um paradigma de pesquisa que tem possibilitado investigar de forma mais precisa a $\mathrm{CE}$ em crianças considerando, simultaneamente, nove componentes que, em função do nível de desenvolvimento, podem ser agrupadas em três fases: uma fase externa (3-6 anos) que envolve a possibilidade de identificar expressões faciais que indicam uma emoção, causadas por fatores externos e a capacidade de recordar uma lembrança passada que possa causar uma emoção; uma fase mental (5-9 anos) que compreende a capacidade de compreender que as emoções são resultantes de crenças e desejos individuais, envolvendo a diferenciação entre estados reais e aparentes, o que é baseado na compreensão de representações mentais; e uma fase reflexiva (8-12 anos) que inclui a possibilidade de identificar que uma pessoa pode sentir mais do que uma emoção, a compreensão 
das emoções morais (como culpa) e o controle mental das emoções (Pons Harris, \& Rosnay, 2004).

Baseados na literatura experimental Pons et al., (2004) construíram o Test of Emotion Comprehension (TEC), validando-o numa amostra de 100 crianças inglesas de três, cinco, nove e onze anos, equitativamente distribuídos por sexo em cada faixa etária. As propriedades psicométricas do TEC foram estudadas, sendo priorizada a análise de estrutura fatorial, da consistência interna do instrumento $(\alpha=.67)$. Também foram conduzidas análises de teste-reteste com três meses $(r(18)=$ $.84)$ e com13 meses $(r(40)=.68)$, sendo controlados os efeitos de género (Pons et al., 2004). O TEC tem sido traduzido em mais de 15 idiomas, existindo atualmente, por exemplo, versões em italiano (Albanese \& Molina, 2008; Farina \& Belacchi, 2014, com consistência interna de $\alpha=.79$ e $\alpha=.76$ respectivamente), alemão (Von Salisch, Haenel, \& Freund, 2013, com $\alpha=.61$ de consistência interna), norueguês (Karstad, Kvello, Wichstrom, \& Berg-Nielsen, 2014; consistência interna de $\alpha=.61, \varphi=.95$ ), quéchua (Tenenbaum, Visscher, Pons, \& Harros, 2004), chinês (Tang et al., 2018), e uma versão portuguesa realizada por Roazzi, Dias, Minervino, Roazzi e Pons $(2008,2009)$ e em Portugal (Franco, Beja, Candeias, \& Santos, 2017, consistência interna de $K R=.72$; Rocha, Roazzi, et al., 2015, estabilidade da escala em três meses $r=.75$ ). Alguns desses estudos também demonstraram que os nove componentes do TEC cumprem os requisitos de uma escala de Guttman ou de Mokken, isto é, que todos os componentes formam uma escala ordinal que pode ser ordenada hierarquicamente de tal forma que a resposta correta a um componente também implica uma resposta correta aos componentes de ordem inferior (Pons et al., 2004; Albanese \& Molina, 2008). Na versão portuguesa, as dimensões do TEC também têm sido avaliadas por meio de análise fatorial confirmatório (Rocha, Roazzi, et al., 2015) e de análises escalonares multidimensionais (Roazzi, Rocha et al., 2015; Rocha, Candeias, Roazzi, \& Silva, 2015; Rocha, Roazzi et al., 2015) confirmando a estrutura teórica do instrumento.

Nos estudos com o TEC têm-se também encontrado correlações positivas entre o TEC e as habilidades de linguagem medidas pelo Test of Receptive Grammar, TORG $(r(78)=.81)$ e $r(76)=.52)$ quando os efeitos da idade e género eram controlados e também uma correlação positiva entre o TEC e a WAIS-III $(r(16)=.63)$ (Tenenbaum et al., 2004; Albanese, Stasio, Chiacchio, Fiorilli, \& Pons, 2010). Por outro lado, também foram identificadas correlações com medidas não verbais de inteligência, como por exemplo a inteligência fluída avaliada pelas Coloured Progressive Matrices (Albanese et al., 2010; De Stasio, Fiorilli, \& Di Chiacchio, 2014; Von Salisch et al., 2013). Esses estudos indicam que a idade e a habilidade verbal estão relacionadas com os componentes da fase externa do TEC, mas a inteligência não verbal apresenta um papel significativo no desenvolvimento dos componentes da fase mental (De Stasio et al., 2014) ou dos resultados globais do TEC (Von Salisch et al., 2013). Outros estudos encontraram associações entre os resultados do TEC e as dificuldades de aprendizagem (Pons et al., 2014), o rendimento académico (Franco et al., 2017; Silva, 2012; Rocha, 2016) e com níveis elevados de queixas somáticas e sintomas ansiosos/deprimidos (Bender, Pons, Harris, Esbjorn, \& Reinholdt-Dunne, 2015; Gobel, Henning, Moller, \& Aschersleben, 2017). O TEC também tem sido utilizado na avaliação de programas de treino de competências sociais e emocionais que incluem uma variedade de matérias (e.g., histórias, livros de figuras, vídeos, jogos), com a discussão dos diferentes componentes da CE (Ornaghi, Brockmeier, \& Grazzani, 2014; Ornaghi, Grazzani, Cherubin, Conte, \& Piralli, 2015; Sprung, Much, Harris, Ebesutani, \& Hofmann, 2015).

A versão portuguesa traduzida e validada por Roazzi et al., $(2008,2009)$ do Test of Emotion Comprebension (TEC) de Pons et al., (2004) está dividido em blocos de histórias em ordem pré-estabelecida, com os seguintes contextos: I (reconhecimento) - o reconhecimento das emoções, baseado nas expressões faciais; II (causas externas) - compreensão das causas externas das emoções; III (desejo) - compreensão do desejo despertado; IV (crenças) - compreensão das emoções baseadas em crenças; V (lembrança) - compreensão do papel da lembrança em circunstâncias de avaliação de estados emocionais; VI (regulação) - compreensão das possibilidades de controlar as experiências emocionais; VII (ocultar) - compreensão da possibilidade de esconder um estado emocional; VIII (misto) - compreensão da existência de múltiplas ou até contraditórias (ambivalentes) respostas emocionais; IX (moralidade) - compreensão de expressões morais. Um ponto é atribuído para cada componente respondido corretamente. Cada criança poderá obter no máximo nove pontos e no mínimo zero (Roazzi et al., 2008).

Em Portugal, existem poucos instrumentos de medida para avaliar a CE em crianças (Franco \& Santos, 2015). A maioria destes instrumentos limitam-se a avaliar apenas os componentes do conhecimento emocional, como por exemplo a versão portuguesa do Affect 
Knowledge Test (Machado et al., 2008) e a Escala de avaliação de conhecimento emocional (EACE, de Alves, Cruz, Duarte, \& Martins, 2008). Dada a importância que a CE reveste para o indivíduo tanto do ponto de vista intrapessoal (devido à sua associação com, por exemplo, sintomas de ansiedade e depressão, e.g., Gobel et al., 2017) como interpessoal (por exemplo, devido a sua capacidade de predizer adequação social e comportamental, Izard et al., 2001; Schultz, Izard, Acherman, \& Youngstrom, 2001), e sendo o TEC um dos pouco instrumentos que permitem observar outros componentes, diversos autores têm estado a estudar algumas das propriedades psicométricas do TEC adaptado para a população brasileira na versão de Roazzi et al., (2008, 2009), adaptada pelos mesmos para o português falado no Portugal (Dâmaso, 2011, Gameiro, 2012; Lima, 2012; Nunes, 2012; Roazzi, Rocha et al., 2015; Rocha, Roazzi et al., 2015; Rocha, Candeias et al., 2015). O objetivo principal deste estudo foi o de estudar as propriedades psicométricas da adaptação da versão portuguesa do Teste de Compreensão Emocional, bem como, os contributos que poderá dar para compreender algumas variáveis do contexto escolar. As perguntas que orientam esta investigação são:

- Qual a fiabilidade e estrutura do TEC para a população portuguesa?

- A CE pode ser um bom preditor do rendimento académico e da retenção escolar (a prática de reter o aluno no mesmo grau por mais um ano escolar)?

\section{Método}

\section{Participantes}

Neste estudo participaram 766 estudantes de 9 escolas de pré-escolar e $1 .^{\circ}$ ciclo do ensino básico (primeiros quatro anos do ensino fundamental em Portugal) da Região Autónoma da Madeira (sete públicas e duas do ensino privado), com idades entre os três e os 13 anos $(M=7.21, D P=1.95 ; 49.7 \%, n=381$, do género feminino). A distribuição por anos de escolaridade foi: $27.7 \%$ do pré-escolar $(n=212), 15 \%$ do $1 .{ }^{\circ}$ ano $(n=115), 17.6 \%$ do $2 .^{\circ}$ ano $(n=135), 15.1 \%$ do $3 .^{\circ}$ ano $(n=116)$ e $24.5 \%$ do $4 .^{\circ}$ ano $(n=188)$.

O nível socioeconómico dos alunos foi determinado a partir de um índice compósito das profissões e habilitações académicas do pai e da mãe, tomando valores entre 1 e 4 (Morais et al., 1993). Considerámos, assim, como estatuto baixo as famílias em que o nível socioeconómico e cultural correspondesse ao intervalo
[1;2] como estatuto médio as famílias no intervalo [2;3] e como estatuto alto as famílias no intervalo [3;4]. Para $28.3 \%(n=217)$ dos estudantes não foi possível determinar o estatuto socioeconómico, 35.2\% apresentam um estatuto socioeconómico baixo $(n=270), 22.1 \%$ um estatuto médio ( $n=169)$, e $14.4 \%$ um estatuto socioeconómico alto $(n=110)$.

Do total de estudantes, foi avaliada a inteligência fluida de 552 alunos $(72.1 \%$ da amostra total). Estes alunos apresentavam idades entre os 4 e os 13 anos $(M=7.17, D P=1.82,50.7 \%, n=280$, do género feminino). A distribuição por ano escolar foi: $24.8 \%$ do pré-escolar $(n=137), 20.8 \%$ do $1 .^{\circ}$ ano $(n=115)$, $24.5 \%$ do $2 .^{\circ}$ ano $(n=135), 8.2 \%$ do $3 .^{\circ}$ ano $(n=45)$ e $21.7 \%$ do $4 .^{\circ}$ ano $(n=120)$.

Para apenas 337 alunos $(43.99 \%$ da amostra total) com idades entre os 6 e os 13 anos $(M=8.21, D P=$ $1.42,47.8 \%, n=161$, do género feminino) tivemos autorização para recolher informação sobre o rendimento académico especificamente as notas de matemática, português e estudo do meio (área para a qual concorrem conceitos e métodos das disciplinas de história, geografia e ciências da natureza). Especificamente são $20.5 \%$ alunos do $1 .^{\circ}$ ano $(n=69), 31.5 \%$ alunos do $2 .^{\circ}$ ano $(n=106), 12.5 \%$ alunos de $3 .^{\circ}$ ano $(n=42)$, e $35.6 \%$ alunos do $4 .^{\circ}$ ano de escolaridade $(n=120)$. Nestes participantes foram identificados 58 alunos $(7.5 \%$ da amostra total) que foram retidos pelo menos uma vez ao longo do seu percurso escolar (isto é, repetiram um ano escolar). Estes alunos apresentam idades compreendidas entre os 8 e os 13 anos de idade $(M=10$, $D P=1.08,31.0 \%, \mathrm{n}=18$, do género feminino), sendo a maioria alunos com nível socioeconómico baixo $(n$ $=40,69.0 \%)$ ou médio $(n=7,12.1 \%)$, não existindo informação sobre o nível socioeconómico para 19\% ( $=11)$ dos alunos retidos. No momento da recolha de dados, os alunos retidos encontravam-se: $1.7 \%$ no $1 .{ }^{\circ}$ ano $(n=1), 22.4 \%$ no $2 .^{\circ}$ ano $(n=13), 27.6 \%$ no $3 .^{\circ}$ ano $(n=16)$ e $48.3 \%$ no $4 .^{\circ}$ ano de escolaridade $(n=28)$.

\section{Instrumentos}

Foi utilizada versão portuguesa traduzida por Roazzi et al., $(2008,2009)$ do Test of Emotion Comprehension (TEC), de Pons et al., (2004), que avalia nove componentes da CE. O processo de tradução e adaptação do instrumento seguiu os critérios internacionalmente aceites para adaptação transcultural de medidas, passando-se pela etapa de tradução e retrotradução, análise semântica e a análise dos juízes (Roazzi et al., 2008, 2009). No estudo piloto, participaram 396 
crianças do Brasil, cujos resultados foram comparados com as respostas dadas pelas crianças britânicas, italianas e quéchuas. Os resultados revelaram similaridades nas respostas das crianças brasileiras e as demais amostras. A discrepância foi maior no componente IV (crenças). Verificou-se, no entanto, similaridades com os resultados obtidos pela amostra italiana (Albanese \& Molina, 2008), sendo apontada a necessidade de recodificar a cotação do componente IV (crenças), sendo aceitas duas respostas ("Feliz" e "Bem") como corretas (Roazzi et al., 2008, 2009).

Neste estudo, foi utilizada a versão computorizada do instrumento (Minervino, Roazzi, Dias, \& Roazzi, 2010). Nessa versão, as histórias são narradas por uma vOz feminina enquanto surgem as ilustrações no ecrã, com a criança a responder clicando na resposta que achar correta. A cotação é feita automaticamente pela aplicação, onde um ponto foi atribuído por cada componente respondido corretamente, utilizando o sistema de cotação da versão inglesa (Pons et al., 2004). Apenas no componente IV (crenças) a cotação foi alterada, tal como sugerido por Roazzi et al., (2009), sendo aceitas as respostas "Feliz" e "Bem" como corretas, uma vez que na cultura portuguesa utiliza-se o termo "estar bem” para dizer que se está feliz. Cada criança pode obter entre zero e nove pontos.

Para avaliar a inteligência fluida das crianças foi utilizada a adaptação portuguesa das Coloured Progressive Matrices (CPM, Simões, 2000), amplamente utilizado na população portuguesa, com elevados índices de fiabilidade $(\alpha=.80)$, estabilidade temporal $(r=.87) \mathrm{e}$ estrutura fatorial consistente (Simões, 2000).

\section{Procedimentos}

A colheita dos dados foi realizada na escola frequentada pelas crianças, com a devida autorização e consentimento informado dos pais, da direção da escola e das próprias crianças, seguindo os princípios éticos da investigação científica da Ordem dos Psicólogos Portugueses (Diário de República, 2011). A aplicação das escalas foi realizada individualmente por um psicólogo treinado no uso dos instrumentos utilizados, demorando aproximadamente 30 minutos. As crianças responderam autonomamente a versão computorizada do TEC, com a supervisão do psicólogo.

Para avaliar o rendimento académico foram recolhidas as notas atribuídas pelos professores aos alunos nas disciplinas de português, matemática e estudo do meio (história, geografia e ciências) no fim do semestre no qual foram administrados os instrumentos. Essas notas apresentam a seguinte interpretação qualitativa com a seguinte correspondência em nota brasileira (Diário da República, 2006): 3 - satisfaz (4,5,6, no Brasil), 4 - satisfaz bem (7, 8, 9, no Brasil), 5 - muito bom ou excelente (10, no Brasil).

\section{Análise de Dados}

Após a colheita, os dados foram introduzidos no software Statistical Package for the Social Sciences (SPSS), versão 24.0 para Windows, utilizado nas diferentes análises. Calcularam-se medidas de tendência central e de dispersão. A consistência interna foi avaliada através do coeficiente de Kuder-Richardson (KR-20) e a fiabilidade teste-reteste ( 9 meses) foi avaliada numa amostra de 76 participantes através do teste de correlação de Pearson (r), para a escala total, e de Cramer ( $V$ ) para cada um dos componentes.

Para analisar a dificuldade dos componentes da CE medidas pelo TEC foi utilizada a prova de Cochran. Para as interrelações entre os nove componentes do TEC, efetuou-se uma Análise da Estrutura de Similaridade (SSA; Guttman, 1991). A SSA é uma técnica estatística da família de técnicas de escalonamentos multidimensionais não paramétrica (MDS, Multidimensional Scaling; Roazzi, 1995; Roazzi, Souza, \& Bilsky, 2015), que possibilita converter distâncias de natureza psicológica em distâncias euclidianas, representadas em um espaço geométrico tendo por base o referencial teórico-metodológico da Teoria das Facetas, em projeções SSA para exame das inter-relações de todas as variáveis do estudo. Sendo os componentes do TEC medidas através de escores dicotómicos, na SSA foi utilizado o coeficiente de Jaccard.

Foram efetuadas análises de regressão múltipla, utilizando o método Enter (fixed-step), para investigar se as variáveis sociodemográficas, a inteligência fluída e a CE eram preditoras do rendimento académico. A fim de determinar a adequação do modelo foram observados os resíduos estandardizados dos diferentes casos (Field, 2011). Para confirmar que não existem casos extremos que pudessem influenciar no modelo, foram observados os valores de Cook's distance, cujos valores para serem considerados adequados devem ser inferiores a 1, e os valores de influência (Leverage), cujos valores deverão ser inferiores ao limite recomendado por Field (2011) $(3(k+1) / n$, onde o k é o número de preditores, e $n$ é o tamanho da amostra).

Para determinar se os modelos podem ser generalizados à população madeirense foram observados os pressupostos de multicolinaeridade, homocedasticidade, 
independência dos erros e normalidade dos erros. O pressuposto da independência dos erros foi analisado através do teste de Durbin-Watson cujo valor deverá estar perto de 2. Finalmente, o pressuposto de normalidade dos erros é confirmado quando os erros apresentam uma distribuição normal (Field, 2011).

\section{Resultados e Discussão}

\section{Estatísticas Descritivas}

Neste estudo, todos os componentes do TEC (cf. Tabela 1.) apresentam valores adequados de assimetria $(S k)$ e curtose $(K u)$, sugerindo uma distribuição normal dos dados (Field, 2011). No entanto, os componentes I (reconhecimento) e II (causas externas) apresentam valores inaceitáveis devido às altas taxas de sucesso. Estes resultados eram esperados, uma vez que de acordo com o desenvolvimento da CE estas dois componentes emergem nos primeiros três anos de vida da criança (Franco \& Santos, 2015), pelo que existe uma elevada taxa de sucesso nesta amostra composta por crianças mais velhas. Por outro lado, Rocha, Roazzi et al., (2015) defendem que, apesar de os valores destes dois componentes serem inaceitáveis do ponto de vista psicométrico, a sua utilização em crianças mais velhas permite-lhes familiarizar-se com os objetivos do teste e o seu conteúdo. As correlações totais entre os itens são relativamente baixas (cf. Tabela 1), especialmente nos componentes IV (crenças) e IX (moralidade), no entanto, a maioria encontra-se no intervalo .15 a .20 , considerado adequado em medidas que avaliam características amplas, como a CE (Antony \& Barlow, 2011).

Os resultados do nosso estudo também indicam que o instrumento TEC apresenta uma consistência interna adequada $(K R-20=.72)$, segundo Cohen (1988). Esse valor de consistência interna é semelhante aos de Albanese e Molina (2008), Farina e Belacchi (2014) e Karstad, Kvello, Wichstrom e Berg-Nielsen (2014), apresentados na introdução. No nosso estudo, a fiabilidade teste-reteste $(9$ meses) foi de $r(74)=.58(p<.001)$, análogos aos resultados encontrados pelos autores com 13 meses de intervalo (Pons \& Harris, 2005), mas mais baixas às encontradas por Rocha, Roazzi et al., (2015) com 3 meses de intervalo, corroborando a estabilidade temporal do instrumento.

\section{Análise da Dificuldade dos Componentes}

Os nossos resultados da comparação da proporção de acertos entre os nove componentes indicam que o grau de dificuldade é diferente entre elas $(Q(8)=$ $1,332.09, p<.001)$. Os resultados das análises de contraste (comparando o número de acertos em cada par de componentes), apresentados na Tabela 2 , revelam os diferentes níveis de dificuldade dos componentes. Os componentes II e I (causas externas e reconhecimento, ambos componentes da fase externa descrita por Pons et al., 2004) apresentam o maior número de acertos e os menores níveis de dificuldade. O componente V (lembrança, também da fase externa) é significativamente mais difícil que o componente I (reconhecimento) e, significativamente, mais fácil que o componente IV

Tabela 1.

Análise Descritiva dos Componentes da Compreensão Emocional

\begin{tabular}{lccccccc}
\hline & $M$ & $D P$ & Min & Max & Sk & Ku & $\begin{array}{c}\text { Correlações } \\
\text { entre itens }\end{array}$ \\
\hline I. Reconhecimento & .92 & .27 & 0 & 1 & -3.18 & 8.13 & .183 \\
II. Causas Externas & .94 & .24 & 0 & 1 & -3.71 & 11.80 & .267 \\
III. Desejo & .44 & .50 & 0 & 1 & 0.23 & -1.95 & .235 \\
IV. Crença & .52 & .50 & 0 & 1 & -0.09 & -2.00 & .117 \\
V. Lembrança & .61 & .49 & 0 & 1 & -0.44 & -1.81 & .182 \\
VI. Regulação & .49 & .50 & 0 & 1 & 0.02 & -2.00 & .314 \\
VII. Ocultar & .41 & .49 & 0 & 1 & 0.36 & -1.87 & .265 \\
VIII. Misto & .48 & .50 & 0 & 1 & 0.10 & -2.00 & .282 \\
IX. Moralidade & .28 & .45 & 0 & 1 & 0.96 & -1.09 & .142 \\
Total TEC & 5.10 & 1.79 & 0 & 9 & -0.11 & -0.64 & \\
\hline
\end{tabular}


(crenças, da fase mental descrita por Pons et al., 2004). Com um nível semelhante de dificuldade estão os componentes IV (crenças), VI e VIII (regulação e misto, ambos da fase reflexiva descrita por Pons et al., (2004), indo ao encontro do estudo de Dâmaso (2011). O componente III (desejo, da fase mental) apresenta uma dificuldade semelhante aos componentes VI (regulação) e VIII (misto), mas é significativamente mais difícil que o componente IV (crenças). O componente VII (ocultar, da fase mental) é semelhante em dificuldade aos componentes III (desejo) e VIII (misto), mas apresenta maior dificuldade que o componente VI (regulação). Finalmente, o componente IX (moralidade, da fase reflexiva) apresenta uma maior dificuldade que qualquer outro componente (cf. Tabela 2).

Dâmaso (2011) encontra uma organização semelhante, agrupando em dois grupos os componentes segundo os sucessos, um primeiro grupo composto (apresentados por ordem dificuldade) pelos componentes I (reconhecimento), II (causas externas), V (lembrança) e III (desejo) e um segundo grupo composto pelos componentes IV (crenças), VIII (misto), VII (ocultar), IX (moralidade) e VI (regulação). A grande diferença entre os nossos resultados e os de Dâmaso centra-se na dificuldade do componente IV (crenças) em relação ao componente III (desejo). No nosso estudo, o componente III apresenta um maior nível de dificuldade que o componente IV (crenças). No entanto, ressalte-se ainda que o estudo de Dâmaso (2011) só abarca crianças com idades compreendidas entre os sete e os dez anos, enquanto o nosso estudo inclui crianças muito mais jovens.
Assim, a organização dos componentes do TEC na nossa amostra apresenta uma estrutura hierárquica coerente com os estudos noutras amostras. A semelhança dos estudos originais na amostra inglesa (Pons et al., 2004) e os estudos em amostras italianas (Albanese \& Molina, 2008), os três componentes da fase externa (I - reconhecimento; II - causas externas e V - lembrança) presentam uma maior percentagem de sucesso (cf. Tabela 1). As capacidades de reconhecer das emoções, de identificar os fatores externos que causam as emoções e de perceber que a lembrança de uma situação passada pode causar uma reação emocional, apresentam um desenvolvimento substancial entre os 4 e os 6 anos (Franco \& Santos, 2015), pelo que no início da escolaridade, a maioria dos alunos já consegue responder corretamente a estes componentes. Porém, quando comparados os nossos resultados com os de outras amostras (e.g., Roazzi et al., 2009), observamos que existe uma maior variabilidade na ordenação hierárquica dos componentes das fases mentais e reflexivas. Estes resultados indicam que nas diversas culturas ocorre alguma variação na dificuldade dos componentes, provavelmente devido as diferenças educacionais e culturais (Belachi \& Faria, 2010; Tenenbaum et al., 2004) e individuais na CE que já são observáveis desde muito cedo (Pons \& Harris, 2005).

\section{Análise da Estrutura de Similaridade}

O coeficiente de alienação encontrado na análise da estrutura de similaridade no nosso estudo apresentou um valor de 0.04 que indica um excelente ajuste dos dados no espaço multidimensional da projeção, com

Tabela 2.

Teste de Cochran cada Par de Componentes da Compreensão Emocional

\begin{tabular}{|c|c|c|c|c|c|c|c|c|}
\hline & I & V & IV & VI & VIII & III & VII & IX \\
\hline II & .02 & $-.33^{* * *}$ & $-.42^{* * *}$ & $-.44^{* * *}$ & $-.46^{* * *}$ & $-.50^{* * *}$ & $-.53^{* * *}$ & $-.65^{* * *}$ \\
\hline I & & $-.32^{* * *}$ & $-.40^{* * *}$ & $-.43^{* * *}$ & $-.45^{* * *}$ & $-.48^{* * *}$ & $-.51^{* * *}$ & $-.64^{* * *}$ \\
\hline V & & & $-.08^{*}$ & $-.11^{* * *}$ & $-.13^{* * *}$ & $.16^{* * *}$ & $-.20^{* * *}$ & $-.32^{* * *}$ \\
\hline IV & & & & .03 & .05 & $.08^{*}$ & $.11^{* * *}$ & $.24^{* * *}$ \\
\hline VI & & & & & -.20 & .05 & $-.08^{*}$ & $-.21^{* * *}$ \\
\hline VIII & & & & & & .03 & .06 & $-.19^{* * *}$ \\
\hline III & & & & & & & -.03 & $-.16^{* * *}$ \\
\hline VII & & & & & & & & $-.13^{* * *}$ \\
\hline
\end{tabular}

Nota. $n=766 ;$ I = Reconhecimento; II = Causas externas; III = Desejo; IV = Crenças; V = Lembrança; VI = Regulação; VII = Ocultar; VIII $=$ Misto; IX $=$ Moralidade.

$* p<.05 ; * * p<.01 ; * * * p<.001$. 
uma partição de tipo polar (angular) dos dados, isto é, seus elementos constituem regiões cuneiformes, de forma circumplex, com limites partindo de uma origem comum.

A análise SSA está apresentada na Figura 1, observando-se a partição do campo dimensional euclidiano da projeção em uma estrutura polar que aponta para as fases da CE de Pons et al., (2004). Em sentido horário, da direita para a esquerda, a estrutura polar apresenta uma primeira região de contiguidade conformada pelos elementos relacionados aos aspectos da fase externa das emoções, isto é, os três componentes II (causas externas), I (reconhecimento) e V (lembrança), nesta ordem. Em seguida, na parte inferior da projeção, há dois componentes da fase mental das emoções: VII (ocultar) e III (desejo); do lado esquerdo da projeção, encontram-se localizados os três componentes da fase reflexiva, isto é, VIII (misto), VI (regulação) e IX (moralidade).

$\mathrm{Na}$ parte superior da projeção encontra-se ainda o item da subescala Mental IV (crenças), um tanto deslocado com os outros dois itens mentais VII (ocultar) e III (desejo). Esse deslocamento ocorreu considerando os dados obtidos por Pons et al., (2004) em uma amostra de crianças inglesas. Pequenas diferenças na estrutura relacional entre os vários componentes têm sido observadas também em outros países, provavelmente, decorrentes de diferenças culturais entre as amostras investigadas e as faixas etárias consideradas. Por exemplo, Albanese et al., (2006) observaram também uma estrutura diferente

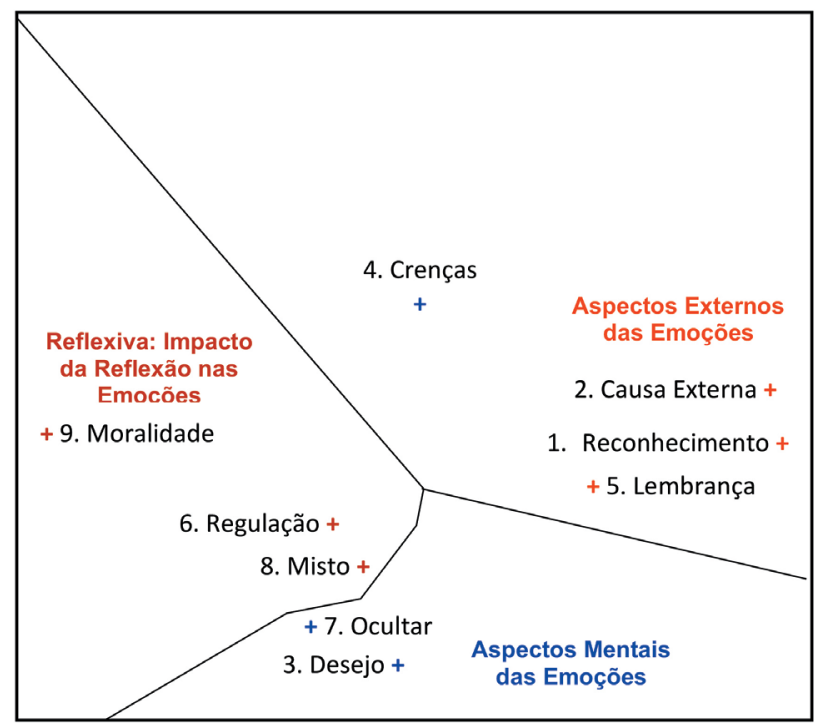

Figura 1. Análise SSA (coeficiente de Jaccard) da interrelação entre os nove componentes do TEC (3d, 1x2, Coeficiente de Alienação 0.045). entre os nove componentes a partir da validação preliminar do TEC em língua italiana, mais especificamente, na subescala da fase externa das emoções em que foram considerados os componentes I (reconhecimento), II (causas externas), e III (desejo), na subescala da fase mental foram agrupados os componentes IV (crenças), V (lembrança) e VII (ocultar, ou distinção emoção aparente/experimentada), enfim, na subescala da fase reflexiva foram incluídos os componentes IX (moralidade), VI (regulação) e VIII (misto). Ulteriores analises com um maior número de participantes poderão delinear mais precisamente a estrutura relacional entre os nove componentes na amostra portuguesa.

\section{Relação entre Compreensão Emocional, Rendimento Académico e Retenção Escolar}

Para investigar a relação estatística entre o rendimento académico e a CE foi realizado um teste de correlação entre as notas e o valor da escala total do TEC, controlando a variável idade. As correlações entre a CE e o rendimento académico são significativas tanto para o português $(r(389)=.24, p<.001)$; como para a matemática: $(r(389)=.25, p<.001)$ e o estudo do meio $(r(389)=.17, p=.001)$.

Foi utilizado o coeficiente de correlação ponto-bisserial para observar a associação entre as notas e as fases da CE (cf. tabela 3). Quando controlada a idade, foram encontradas relações pequenas, mas significativas, entre as notas de português, matemática e o estudo do meio e as fases mental e reflexiva.

Quando controlada a idade, foram encontradas relações pequenas, mas significativas entre as notas de português, matemática e estudo do meio nos componentes VI (regulação), VII (ocultar) e VIII (misto). O componente IV (crenças) também está relacionado com a matemática e o estudo do meio, quando considerados como corretas duas respostas. Pode inferir-se que, quanto maior a compreensão da regulação das emoções, das emoções ambivalentes e da possibilidade de esconder uma emoção melhor é o rendimento dos alunos nas disciplinas de português, matemática e estudo do meio.

No que se refere à retenção escolar, observamos que quando controlada a idade, existe uma correlação negativa $(r(763)=.08, p=.034)$. Assim, uma melhor CE está associada aos alunos que não repetiram nenhum ano escolar. Existem, também, associações significativas negativas para a fase reflexiva (cf. Tabela 3). Pode inferir-se que a pertença à fase reflexiva está associada a uma menor retenção. Igualmente encontramos 
Tabela 3.

Correlações Parciais de Ponto-Bisserial e de Cramer entre Compreensão Emocional, Rendimento Académico e Retenção Escolar

\begin{tabular}{lcccc}
\hline & Português & Matemática & Estudo do Meio & Retenção escolar \\
\hline Externa & .01 & .05 & -.02 & -.01 \\
Mental & $.18^{* * *}$ & $.18^{* * *}$ & $.13^{*}$ & -.02 \\
Reflexiva & $.23^{* * *}$ & $.19^{* * *}$ & $.14^{* *}$ & $-.08^{*}$ \\
I. Reconhecimento & -.03 & -.02 & -.04 & -.01 \\
II. Causas Externas & .06 & .111 & .04 & -.03 \\
III. Desejo & .07 & .09 & -.00 & $-.08^{*}$ \\
IV. Crenças & .10 & $.12^{*}$ & $.13^{*}$ & -.03 \\
V. Lembrança & .01 & .02 & -.04 & -.03 \\
VI. Regulação & $.21^{* * *}$ & $.19^{* * *}$ & $.12^{*}$ & -.02 \\
VII. Ocultar & $.16^{* *}$ & $.15^{* *}$ & $.15^{* *}$ & .01 \\
VIII. Misto & $.19^{* * *}$ & $.22^{* * *}$ & $.17^{* *}$ & $-.10^{* *}$ \\
IX. Moralidade & .02 & -.04 & .01 & -.02 \\
\hline
\end{tabular}

Nota. $n=395$. Variável de controlo: Idade em anos.

$* p<.05, * * p<.01, * * * p<.001$.

associações negativas entre os componentes III (desejo) e VIII (misto) e a retenção escolar, indicando que uma melhor compreensão da influência do desejo nas emoções e das emoções mistas pode estar associada a uma menor retenção.

Compreensão Emocional como Preditora do Rendimento Académico Com base nos resultados das correlações, foi efetuada uma análise de regressão múltipla (método Enter) para investigar se os componentes VI (regulação), VII (ocultar) e VIII (misto) eram preditores do rendimento académico em Português, e se os componentes IV (crenças), VI (regulação), VII (ocultar) e VIII (misto) eram preditores do rendimento académico em Matemática e em Estudo do Meio.

Os resultados indicam que os melhores preditores do rendimento académico em Português são o nível socioeconómico $(b=0.344, t=4.62, p<.001)$ e a inteligência fluida $(b=0.025, t=4.03, p<.001)$, seguidos pela idade $(b=-0.10, t=-2.21, p=.028)$, a compreensão do componente VI (regulação; $b=0.275, t=2.31, p$ $=.022$ ), e a compreensão do componente VIII (misto; $b=0.273, t=2.33, p=.021)$. Os resultados apresentados na tabela 4 indicam que quanto maior o nível socioeconómico, a inteligência fluida e a compreensão dos componentes VI (regulação) e VIII (misto), melhor o rendimento dos alunos em português. Os resultados também indicam que quanto maior a idade, pior é o rendimento dos alunos. A combinação das variáveis explica $22.1 \%$ da variância, sendo que os componentes da CE explicam 3.8\% da variância no rendimento académico em português (cf. Tabela 5). Assim, parte da variabilidade dos resultados académicos dos alunos em português é explicada pelas suas capacidades de CE, da sua inteligência fluída e a sua idade.

$\mathrm{Na}$ matemática, os melhores preditores do rendimento académico em matemática são a inteligência fluida $(b=0.034, t=5.06, p<.001)$ e a idade $(b=-0.206$, $t=-4.37, p<.001)$, seguidos pelo nível socioeconómico $(b=0.342, t=4.28, p<.001)$, e a compreensão do componente VIII (misto; $b=0.365, t=2.90, p=.004$ ). Os componentes IV (crenças), VI (regulação) e VII (ocultar) não parecem contribuir significativamente. Assim, e como apresentado na tabela 4 , quanto maior a inteligência fluida, o nível socioeconómico e a compreensão das emoções mistas, melhor o rendimento dos alunos em matemática; e quanto maior a idade, pior é o rendimento dos alunos. A combinação destas variáveis explica $27.2 \%$ da variância, sendo que os componentes da CE explicam 3.2\% da variância no rendimento académico em matemática (cf. Tabela 5).

Finalmente, os resultados indicam que os melhores preditores do rendimento académico em estudo do meio são a idade $(b=-0.245, t=-6.14, p<.001)$ e o nível socioeconómico $(b=0.321, t=4.74, p<.001)$, seguidos pela compreensão do componente VIII 
(misto; $b=0.245, t=2.30, p=.022$ ) e pela inteligência fluida ( $b=0.012, t=2.16, p=.032$ ). O componente VII (ocultar) apresenta uma contribuição marginalmente significativa $(b=0.208, t=1.92, p=.056)$. Dito de outra forma, quanto maior o nível socioeconómico, a compreensão do componente VIII (misto) e a inteligência fluida, melhor o rendimento dos alunos na disciplina de estudo do meio; e quanto maior a idade, pior é o rendimento dos alunos (cf. Tabela 4). As variáveis explicam $25.1 \%$ da variância, sendo que os componentes da CE explicam 3\% da variância no rendimento académico em estudo do meio (cf. Tabela 5).

Tabela 4.

Coeficientes Estandardizados dos Modelos de Regressão Múltipla para os Preditores do Rendimento Académico

\begin{tabular}{|c|c|c|c|c|c|c|c|c|c|}
\hline \multirow{2}{*}{ Modelos } & $\begin{array}{c}\beta \\
\text { Português }\end{array}$ & $\begin{array}{c}\beta \\
\text { Matemática }\end{array}$ & $\begin{array}{c}\beta \\
\text { Estudo do Meio }\end{array}$ & & & & & & \\
\hline & I & II & III & I & II & III & I & II & III \\
\hline Constante & 2.50 & 0.98 & 1.45 & 3.20 & 1.25 & 1.65 & 4.08 & 3.31 & 3.57 \\
\hline Género & -.02 & -.03 & -.01 & .06 & .05 & .07 & .08 & .08 & .10 \\
\hline Nível socioeconómico & $.33^{* * *}$ & $.27 * * *$ & $.24 * * *$ & $.31 * * *$ & $.24 * * *$ & $.22 * * *$ & $.30 * * *$ & $.27 * * *$ & $.25^{* * *}$ \\
\hline Idade & -.05 & -.04 & $-.12^{*}$ & $-.17 * *$ & $-.17^{* *}$ & $-.23 * * *$ & $-.27 * * *$ & $-.27 * * *$ & $-.33 * * *$ \\
\hline MPCR & & $.27 * * *$ & $.21 * * *$ & & $.31 * * *$ & $.26^{* * *}$ & & $.14^{* *}$ & $.11^{*}$ \\
\hline IV. Crenças & & & - & & & .05 & & & .07 \\
\hline VI. Regulação & & & $.12^{*}$ & & & .07 & & & .01 \\
\hline VII. Ocultar & & & .09 & & & .06 & & & .10 \\
\hline VIII. Misto & & & $.12^{*}$ & & & $.15^{* *}$ & & & $.12^{*}$ \\
\hline
\end{tabular}

Nota. $n=321$; Modelo I = Variáveis sociodemográficas (Género, nível socioeconómico, idade); Modelo II = Inteligência fluída (MPCR); Modelo III = Compreensão emocional (Componentes IV - crenças, VI - regulação, VII - ocultar e VIII - misto).

$* p<.05 ; * * p<.01 ; * * * p<.001$.

Tabela 5.

Modelos de Regressão Múltipla dos Preditores do Rendimento Académico

\begin{tabular}{llccccc}
\hline $\begin{array}{c}\text { Variável } \\
\text { dependente }\end{array}$ & \multicolumn{1}{c}{ Modelos } & $R$ & $R^{2}$ & $\Delta R^{2}$ & $F$ & $\Delta F$ \\
\hline Português & I - Variáveis Sociodemográficas & .341 & .116 & .108 & $13.90^{* * *}$ & $13.90^{* * *}$ \\
& & & & & & \\
& II - Inteligência fluída & .428 & .183 & .173 & $17.73^{* * *}$ & $25.95^{* * *}$ \\
& III - Compreensão Emocional & .470 & .221 & .203 & $12.67^{* * *}$ & $5.02^{* *}$ \\
Matemática & I - Variáveis Sociodemográficas & .388 & .151 & .143 & $18.75^{* * *}$ & $18.75^{* * *}$ \\
& II - Inteligência fluída & .490 & .240 & .230 & $24.95^{* * *}$ & $37.12^{* * *}$ \\
& III - Compreensão Emocional & .521 & .272 & .253 & $14.57^{* * *}$ & $3.42^{* *}$ \\
Estudo do Meio & I - Variáveis Sociodemográficas & .450 & .202 & .194 & $26.67^{* * *}$ & $26.67^{* * *}$ \\
& II - Inteligência fluída & .471 & .222 & .212 & $22.41^{* * *}$ & $7.88^{* *}$ \\
& III - Compreensão Emocional & .501 & .251 & .232 & $13.05^{* * *}$ & $3.09^{*}$ \\
\hline
\end{tabular}

Nota. R: coeficiente de correlação; $R^{2}$ : coeficiente de determinação; $\Delta R^{2}$ : coeficiente de determinação ajustado; $F$ : estatístico da regressão; $\Delta F$ : estatísticos de alteração do modelo; $n=321$.

$* p<.050 ; * * p<.010 ; * * * p<.001$.

Psico-USF, Bragança Paulista, v. 25, n. 2, p. 247-260, abr./jun. 2020 
Todos os modelos apresentam-se como adequados e generalizáveis à população madeirense, uma vez que a amostra em estudo não apresenta casos extremos que possam influenciar o modelo e os pressupostos de multicolinearidade, independência dos erros, de homocedasticidade e de normalidade dos erros foram cumpridos.

Como vários estudos têm vindo a mostrar (Downey, Lomas, Billings, Hansen, \& Stough, 2014; Izard et al., 2001; Rhoades, Warren, Domitrovich, \& Greenberg, 2011; Torres, Domitrovich, \& Bierman, 2015), a CE é um preditor pobre do rendimento académico. No entanto, numa meta-análise feita por Voltmer e von Salisch (2017), fica claro que esta relação pode ter efeitos moderados $(r=.32$,) e que a força da correlação depende de vários fatores, especialmente do estatuto socioeconómico do aluno, o que vai ao encontro dos nossos resultados. Outra variável que é um importante mediador entre a CE e o rendimento académico são as competências sociais (Caprara, Barbaranellu, Pastorelli, Bandura, \& Zimbardo, 2000; Denham et al., 2012; Franco et al., 2017; Izard et al., 2001; Oberle, Schonert-Reichl, Hertzman, \& Zumbo, 2014). Na medida em que a CE permite uma melhor leitura emocional das situações (nomeadamente das relações professor-aluno) facilita o relacionamento, as atitudes e os comportamentos em sala de aula, dirigindo melhor a atenção do aluno para as tarefas e os desempenhos esperados.

\section{Considerações Finais}

O objetivo principal deste estudo foi o de estudar as propriedades psicométricas da adaptação da versão portuguesa do TEC, bem como, os contributos que poderão dar para compreender algumas variáveis do contexto escolar. Os nossos resultados permitem respaldar as muitas vantagens deste instrumento. Em primeiro lugar, o instrumento não se restringe a um ou dois aspetos da CE como o EACE ou o Affect Knowledge Test, mas permite avaliar nove componentes, todos eles bem documentados na literatura experimental (Franco \& Santos, 2015). Em segundo lugar, os nossos resultados demonstram que é um instrumento com valores aceitáveis de consistência interna e estabilidade, o que faz com que se possa continuar a aplicar este instrumento para medir a CE, com alguma confiança. Em terceiro lugar, a estrutura do TEC serve para medir três fases diferentes do desenvolvimento da CE: a fase mental, a fase externa e a fase reflexiva, sendo ainda necessário afinar os componentes do TEC que compõem cada uma dessas fases, pois uma vez mais se fez sentir a influência de possíveis fatores culturais. Pese a estas limitações, o instrumento demonstra sensibilidade suficiente para detectar diferenças individuais no desenvolvimento da CE. Em quarto lugar, a capacidade explicativa que a CE tem do rendimento académico é relevante, apesar de ser baixa e dependente de várias variáveis intermédias (e.g. idade, inteligência fluida e nível socioeconómico). Por último, a versão computadorizada é de fácil aplicação e muito atrativa para as crianças permitindo, num curto período de tempo, obter informação relevante em relação à CE das crianças.

Entre as limitações deste estudo está o cariz correlacional dos resultados é insuficiente para explicar uma relação de causalidade entre a CE e o rendimento académico. Da mesma forma, o caráter transversal do estudo impede estabelecer uma relação de ordem temporal. Futuros estudos deverão desenvolver estudos longitudinais que permitam controlar o desempenho prévio.

Tendo em conta a grande amplitude de idades das crianças deste estudo, poderá ser importante num futuro fazer uma análise do funcionamento do TEC por grupos etários, quer para verificar se há organizações diferentes dos componentes do TEC entre estes quer para mostrar se diferenças no funcionamento da CE nos diferentes grupos etários explicam diferenças no rendimento académico. Seria igualmente interessante fazer comparações com crianças de diferentes etnias e culturas.

\section{Referências}

Albanese, O., \& Molina, P. (2008). Lo sviluppo della comprensione delle emozioni e la sua valutazione. La standardizzazione italiana del Test di Comprensione delle Emozioni (TEC). Milano: Edizioni Unicopli.

Albanese, O., De Stasio, S., Chicchio, C., Fiorilli, C., \& Pons, F. (2010). Emotion Comprehension: The Impact of Nonverbal Intelligence. The journal of genetic psychology, 171(2), 101-115. doi:10.1080/00221320903548084

Albanese, O., Grazzani, I., Molina, P., Antoniotti, C., Arati, L., Farina, E., \& Pons, F. (2006). La comprensione delle emozioni nei bambini: dati preliminari del progetto italiano di validazione del Test of Emotion Comprehension. In O. Albanese, 
L. Lafortune, M. F. Daniel, P. A. Doudin, \& F. Pons (Eds.), Competenza emotiva tra psicologia ed educazione (pp. 35-47). Milano: Franco Angeli.

Alves, D., Cruz, O., Duarte, C., \& Martins, R. (2008). Escala de Avaliação do conhecimento Emocional (EACE). In A.P. Noronha, C. Machado, L. S. Almeida, M. Gonçalves, S. Martins \& V. Ramalho (Eds.), Actas da XIII Conferência Internacional Avaliação Psicológica: Formas e Contextos (CD). Braga: Psiquilibrios.

Antony, M. M., \& Barlow, D. H. (2011). Handbook of assessment and treatment planning for psychological disorders $\left(2^{\text {nd }}\right.$ Ed. $)$. New York, NY: Guilford Press.

Bender, P. K., Pons, F., Harris, P. L., Esbjorn, B. H., \& Reinholdt-Dunne, M. L. (2015). Emotion understanding in clinically anxious children: a preliminary investigation. Frontiers of Psychology, 6, 1916. doi:103389/fpsyg.2015.01916

Caprara, G. V., Barbaranelli, C., Pastorelli, C., Bandura, A., \& Zimbardo, P. G. (2000). Prosocial Foundations of Children's Academic Achievement. Psychological Science, 11(4), 302-306. doi:10.1111/1467-9280.00260

Cohen, J. (1988). Statistical powver analysis for the behavioral sciences (2nd ed.). Hillsdale: Erlbaum.

Dâmaso, P. P. (2011). Especificidade na diversidade: A compreensão emocional em crianças com dificuldades de aprendizagem (Dissertação de mestrado). ISCTE-IUL, Lisboa. Recuperado de http://hdl.handle. net/10071/4133

De Stasio, S., Fiorilli, C., \& Di Chiacchio, C. (2014). Effects of verbal ability and fluid intelligence on children's emotion understanding. International Journal of Psychology, 49, 409-414. doi:10.1002/ ijop.12032

Denham, S. A., Bassett, H. H., Way, E., Mincic, M., Zinsser, K., \& Graling, K. (2012). Preschoolers' emotion knowledge: Self-regulatory foundations, and predictions of early school success. Cognition and Emotion, 26(4), 667-679. doi:10.1080/0269993 1.2011 .602049

Diário da República. $1 .{ }^{\text {a }}$ Série $133 / 1$ do dia 12 de julho (2006).

Diário da República. 2. Série 246/2 do dia 26 de Dezembro (2011).
Djambazova-Popordanoska, S. (2016). Implications of emotion regulation on young children's emotional wellbeing and educational achievement. Educational Review, 68(4), 497-515. doi:10.1080/00131911.201 6.1144559

Downey L. A., Lomas J., Billings C., Hansen K., \& Stough C. (2014). Scholastic success: Fluid intelligence, personality, and emotional intelligence. Canadian Journal of School Psychology, 29, 40-53. doi:10.1177/0829573513505411

Farina, E., \& Belacchi, C. (2014). The relationship between emotional competence and hostile/prosocial behavior in Albanian preschoolers: An exploratory study. School Psychology International, 35(5), 475-484. doi:10.1177/0143034313511011

Field, A. (2011). Discovering statistics using SPSS. London: Sage.

Franco, M. G., Beja, M. J., Candeias, A., \& Santos, N. (2017). Emotion understanding, Social competence and school achievement in children from primary school in Portugal. Frontiers in Psychology, 8, 1376. doi.10.3389/fpsyg.2017.01376

Franco, M. G., \& Santos, N. N. (2015). Desenvolvimento da compreensão emocional. Psicologia: Teoria e Pesquisa, 31(3), 334-348. doi:10.1590/0102-37722015032099339348

Gameiro, S. S. (2012). A compreensão das emocões em crianças dos 6 aos 8 anos - Estudo de adaptação portuguesa do TEC (Dissertação de mestrado). Universidade de Coimbra. Recuperado de http://hdl.handle. net/10316/22659

Gobel, A., Henning, A., Moller, C., \& Aschersleben, G. (2017). The relationship between emotion comprehension and internalizing and externalizing behavior in 7- to 10-year-old children. Frontiers in Psychology, 7, 1917. doi:10.3389/fpsyg.2016.01917

Guttman, L. (1991). Louis Guttman: In memoriam - Chapters from an unfinished textbook on facet theory. Jerusalém: The Israel Academy of Sciences and Humanities.

Izard, C., Fine, S., Schultz, D., Mostow, A., Ackerman, B., \& Youngstrom, E. (2001). Emotion Knowledge as a predictor of social behavior and academic competence in children at risk. Psychological Science, 12(1), 18-23. doi:10.1111/1467-9280.00304

Karstad, S. B., Kvello, O., Wichstrom, L., \& BergNielsen, T. S. (2014). What do parents know about 
their children's comprehension of emotions? Accuracy of parental estimates in a com- munity sample of pre-schoolers. Child: Care, Health and Development, 40, 346-353. doi:10.1111/cch.12071

Lima, A. C. (2012). A compreensão das emoções em crianças dos 4 aos 5 anos - Estudo de adaptação portuguesa do TEC (Dissertação de mestrado). Universidade de Coimbra. Recuperado de http://hdl.handle. net $/ 10316 / 23267$

Machado, P., Veríssimo, M., Torres, N., Peceguina, I, Santos, A. J., \& Rolão, T. (2008). Relações entre o conhecimento das emoções, as competências académicas, as competências sociais e a aceitação entre pares. Análise Psicológica, 26(3), 463-478. Recuperado de http://hdl.handle.net/10400.12/70

Minervino, C. M., Roazzi, A., Dias, M. G., \& Roazzi, M. (2010). Versão computadorizada do teste de compreensão das emoções. In M. C. R. A. Joly, \& C. T. Reppold (Eds.), Estudo de testes informatizados para a avaliação psicológica. (E-book, pp. 109-123). São Paulo: Cada do Psicólogo.

Morais, A., Neves, I., Medeiros, A., Peneda, D., Fernanda, F., \& Antunes, H. (1993). Socialização primária e prática pedagógica (Vol. II). Lisboa: Fundação Calouste Gulbenkian.

Nunes, J. (2012). A compreensão das emoções em crianças dos 9 aos 11 anos (Dissertação de mestrado). Universidade de Coimbra. Recuperado de http://hdl. handle.net/10316/22441

Oberle, E., Schonert-Reichl, K. A., Hertzman, C., \& Zumbo, B. D. (2014). Social - emotional competencies make the grade: Predicting academic success in early adolescence. Journal of Applied Developmental Psychology, 35(3), 138-147. doi:10.1016/j. appdev.2014.02.004

Ornaghi, V., Brockmeier, J., \& Grazzani, I. (2014). Enhancing social cognition by training children in emotion understanding: a primary school study. Journal of Experimental Child Psychology, 119, 26-39. doi:10.1016/j.jecp.2013.10.005

Ornaghi, V., Grazzani, I., Cherubin, E., Conte, E., \& Piralli, F. (2015). 'Let's talk about emotions'. The effect of converstional treaining on preschoolers' emotion comprehension and prosocial orientation. Social development, 24, 166-183. doi:10.1111/ sode.12091
Pons, F., \& Harris, P. L. (2005). Longitudinal change and longitudinal stability of individual differences in children's emotion understanding. Cognition and Emotion, 19(8), 1158-1174. doi: $10.1080 / 02699930500282108$

Pons, F., Harris, P. L., \& de Rosnay, M. (2004). Emotion comprehension between 3 and 11 years: Developmental periods and hierarchical organization. European Journal of Developmental Psychology, 1(2), 127-152. doi:10.1080/17405620344000022

Rhoades B. L., Warren H. K., Domitrovich C. E., \& Greenberg M. T. (2011). Examining the link between preschool social-emotional competence and first grade academic achievement: the role of attention skills. Early Childhood Research Quarterly, 26, 182-191. doi:10.1016/j.ecresq.2010.07.003

Roazzi, A. (1995). Categorização, formação de conceitos e processos de construção de mundo: Procedimento de classificações múltiplas para o estudo de sistemas conceituais e sua forma de análise através de métodos de análise multidimensionais. Cadernos de Psicologia, 1, 1-27.

Roazzi, A., Dias, M. G., Minervino, C. M., Roazzi, M., \& Pons, F. (2008). Compreensão das emoções em crianças: Estudo transcultural sobre a validação do Teste de Compreensão da Emoção TEC (Test of Emotion Comprehension). Em A. Noronha, C. Machado, L. Almeida, M. Gonçalves, S. Martins \& V. Ramalho (Eds.), Actas da XIII Conferência Internacional de Avaliação Psicológica: Forma e Contextos (pp. 1781-1795). Braga: Psiquilibrios edições.

Roazzi, A., Dias, M. G., Minervino, C. M., Roazzi, M., \& Pons, F. (2009). Children's comprehension of emotion: A cross-cultural investigation. In D. Elizur \& E. Yaniv (Eds.), Theory Construction and Multivariate Analysis: Applications of Facet Approach (pp. 83-102). Jerusalem: FTA. doi:10.13140/ RG.2.1.2790.3600

Roazzi, A., Rocha, A. A., Candeias, A. A., Silva, A. L., Minervino, C. A., Roazzi, M. M., \& Pons, F. (2015). Social competence and emotional comprehension: How are they related in children? In A. Roazzi, B. C. de Souza, \& W. Bilsky (Eds.), Facet Theory: Searching for Structure in Complex Social, Cultural and Psychological Phenomena (pp. 267-283). Recife: Editora UFPE. doi:10.13140/ RG.2.1.3374.9524 
Roazzi, A., Souza, B. C., \& Bilsky, W. (2015). Facet Theory: Searching for structure in complex social, cultural and psychological phenomena. Recife: Editora Universitária da UFPE.

Rocha, A. M. A. (2016). Compreensão e regulação das emoçoes: suas relações com a eficácia na interação social em crianças. (Tese de doutoramento). Universidade de Lisboa e Universidade de Coimbra.

Rocha, A. A., Candeias, A. A., Roazzi, A., \& Silva, A. L. (2015). Socially in Action-Peers (SAp): Validation by Means of Confirmatory Factor Analysis (CFA) and Similarity Structure Analysis (SSA). In A. Roazzi, B. C. de Souza, \& W. Bilsky (Eds.), Facet Theory: Searching for Structure in Complex Social, Cultural and Psychological Phenomena (pp. 85-106). Recife: Editora UFPE. doi:10.13140/RG.2.1.1671.0165

Rocha, A. A., Roazzi, A., Silva, A. L., Candeias, A. A., Minervino, C. A., Roazzi, M. M., \& Pons, F. (2015). Test of Emotion Comprehension: Exploring the underlying structure through Confirmatory Factor Analysis and Similarity Structure Analysis. Em A. Roazzi, B. C. de Souza, \& W. Bilsky (Eds.), Facet Theory: Searching for Structure in Complex Social, Cultural and Psychological Phenomena (pp. 66-84). Recife: Editora UFPE. doi:10.13140/RG.2.1.2457.4483

Schultz, D., Izard, C., Ackerman, B., \& Youngstrom, E. (2001). Emotion knowledge in economically disadvantaged children: self-regulatory antecedents and relations to social difficulties and withdrawal. Development and Psychopathology, 13(1), 53-67. doi:10.1017/S0954579401001043

Silva, E. P. (2012). A relação entre inteligência emocional e o rendimento escolar em crianças do $1 .^{\circ}$ ciclo do ensino básico da R.A.M. (Dissertação de Mestrado). Universidade da Madeira, Funchal, recuperado de http://hdl.handle.net/10400.13/687

Simões, M. (2000). Investigações no âmbito da aferição nacional do teste das matrizes progressivas coloridas de Raven (MPCR). Lisboa: Fundação Calouste Gulbenkian.

Sprung, M., Munch, H. M., Harris, P. L., Ebesutani, C., \& Hoffman, S. G. (2015). Childrn's emotion understanding: A meta-analysis of training studies, Developmental Review, 27, 41-65. doi:10.1016/j. dr.2015.05.001

Tenenbaum, H., Visscher, P., Pons, F., \& Harris, P. (2004). Emotional undestanding in Quechua children from an agro-pastoralist village. International Journal of Behavioral Development, 28(5), 471-478. doi: 10.1080/01650250444000225

Torres, M. M., Domitrovich, C. E., \& Bierman, K. L. (2015). Preschool interpersonal relationships predict kindergarten achievement: mediated by gains in emotion knowledge. Journal of Applied Developmental Psychology, 39, 44-52. doi:10.1016/j. appdev.2015.04.008

Voltmer, K., \& von Salisch, M. (2017). Three metaanalyses of children's emotion knowledge and their school success. Learning and Individual Differences, 59, 107-118. doi:10.1016/j.lindif.2017.08.006

Von Salisch, M., Haenel, M., \& Freund, P. A. (2013). Emotion understanding and cognitive abilities in young children. Learning and Individual Differences, 26, 15-19. doi:10.1016/j.lindif.2013.04.001

Recebido em: 04/06/2018

Reformulado em: 26/02/2019 
Sobre os autores:

Maria da Gloria Franco é doutora em Psicologia da Educação (Universidade da Madeira, Portugal, 2003) e professora auxiliar na Faculdade de Artes e Humanidades da Universidade da Madeira, onde leciona atualmente Psicologia da Educação e Avaliação Psicológica. É responsável pelo Mestrado em Psicologia da Educação na Universidade da Madeira. Seus interesses de pesquisa incluem: inteligência emocional, habilidades socioemocionais, avaliação psicológica, programas psico-educacionais, estudos sobre violência.

ORCID: https://orcid.org/0000-0001-8634-9120

E-mail: gloria@uma.pt

António Roazzi é doutor (D. Phil.) em Psicologia do Desenvolvimento Cognitivo pela University of Oxford obtido em 1988, possui também título de "Dottore" em Psicologia Aplicada pela Universitá degli Studi di Roma "La Sapienza". É professor titular do Dep. de Psicologia da Universidade Federal de Pernambuco e pesquisador Nível 1A do CNPq. Atua, principalmente, nos seguintes temas: avaliação psicológica, autoconsciência e estados ampliados de consciência, psicologia do desenvolvimento sociocognitivo, aprendizagem, lógica mental, teoria da mente, percepção de risco, gestão de pessoas, apego, violência urbana e o componente ambiental ressaltando os processos cognitivos, sociais e imagéticos de seus habitantes, entre outros.

ORCID: https://orcid.org/0000-0001-6411-2763

E-mail: roazzi@gmail.com

Natalie Nóbrega Santos é cum laude em Educação de Infância e mestre em Psicologia da Educação, colabora em diversos projetos de Educação Básica e Pré-escolar, especificamente na área do desenvolvimento de competências socioemocionais em alunos e professores, nas componentes afetivas da aprendizagem e o seu impacto no desempenho académico e nas conceções de avaliação dos professores.

ORCID: https://orcid.org/0000-0002-4973-9311

E-mail: natalie_nobrega_santos@hotmail.com

\section{Contato com os autores:}

Maria da Glória Franco

Campus Universitário da Penteada

Funchal, Portugal

9020-105 